\title{
Clinical Characteristics of Patients with Original Clinical Characteristics of Patients with Article Medically Unexplained Chronic Widespread Pain: A Primary Care Center Study
}

\author{
Kye Hwa Lee, Cheol Hwan Kim*, Ho Cheol Shin, Eun Ju Sung
}

Department of Family Medicine, Kangbuk Samsung Hospital, Sungkyunkwan University School of Medicine, Seoul, Korea

Background: Chronic widespread pain (CWP) is known as a common symptom of several organic and psychological disorders. Although medically unexplained CWP (MUE) has lots of clinical distress symptoms, there were no distinct symptoms or signs. Therefore, we conducted this study to investigate clinical distress symptoms of MUE distinct from those of medically explained CWP (ME).

Methods: One hundred nine patients with CWP were enrolled in the study. We classified the study subjects into three groups depending on their medical problems associated with CWP: organic group (ORG), psychological group (PSY), and MUE. All subjects were asked to fill out self-report questionnaires consisting of clinical distress scales including the Korean version of the Fibromyalgia Impact Questionnaire (FIQ-K), fatigue scale, depression scale, and stress scale. And physicians examined 18 tender points over their entire body of the subjects.

Results: MUE patients had higher FIQ-K and fatigue severity scores than ORG patients (all P < 0.05). The average number of tender points were 11.33 in MUE patients, 6.48 in ORG patients and 5.02 in PSY patients and statistically significant (P $<0.0001$ ). There were no statistically different factors between MUE and PSY patients with exception for the number of tender points. Depressive symptom was the highest in PSY patients but not statistically different from MUE patients.

Conclusion: MUE patients had higher physical impairments, fatigue severity and more number of tender points than ORG patients, but had no different clinical characteristics from PSY patients except for the number of tender points.

Keywords: Chronic Pain; Fibromyalgia Syndrome; Chronic Fatigue Syndrome

\section{INTRODUCTION}

Received: May 4, 2011, Accepted: June 16, 2011

*Corresponding Author: Cheol Hwan Kim

Tel: 82-2-2001-2281, Fax: 82-2-2001-1260

E-mail:kchosh@nate.com

Korean Journal of Family Medicine

Copyright (C) 2011 The Korean Academy of Family Medicine

(c) This is an open-access article distributed under the terms of the Creative Commons Attribution Non-Commercial License (http://creativecommons.org/licenses/by-nc/3.0) which permits unrestricted noncommercial use, distribution, and reproduction in any medium, provided the original work is properly cited.
Chronic widespread pain (CWP) is defined as a pain on four quadrants of the body as well as the axial skeleton persisting over 3 months. The prevalence of CWP occurs in 10-14\% of the general population and is more common in women than men. ${ }^{1,2)}$ CWP is a long-lasting condition and worsens patients' quality of life. ${ }^{3-5)}$ Numerous studies have been performed on patients with fibromyalgia syndrome (FMS) and CWP since Wolfe et al. ${ }^{6}$ ) suggested a criteria for FMS (American College of Rheumatology, ACR) in 1990. CWP has distinct symptoms for specific organic and psychological disorders although some are medically unexplained in clinical practice. ${ }^{7)}$ FMS is not a 
specific disease but a syndrome of several medically unexplained symptoms such as chronic pain, chronic fatigue, sleep disturbances and mood disorders. ${ }^{8,9)}$ CWP patients are difficult for the physician to treat and there are few studies for distinct clinical characteristics of medically unexplained CWP. This study was designed to investigate the differential clinical characteristics between medically unexplained and explained (organic or psychological) CWP patients.

\section{METHODS}

\section{Study Participants}

This study was conducted in a department of family medicine at a university hospital in Seoul, Korea. From April 12 to July 15 in 2010, patients who agreed to participate in the study were interviewed to assess their pain sites and duration by two trained nurses. This study protocol was approved by the ethics committee of the Kangbuk Samsung Hospital, Sungkyunkwan University School of Medicine (IRB No. KBS10037), and informed consent was obtained from each subject. Total 109 CWP patients were chosen as the final subjects of this study. CWP was defined as follows: pain sites on both sides of the body, above and below the waist as well as the axial skeleton of the body, which has persisted more than 3 months. Patients who didn't respond to a questionnaire appropriately were excluded. Physicians later rechecked the pain sites using a second interview. Further, all subjects were asked to fill out a self-report questionnaire.

\section{Questionnaires}

The questionnaire were designed as self-reporting and consisted of socio-demographic status, habitual status (smoking and alcohol) and clinical distress scales. Socio-demographic factors were age, sex, marital status, level of education and current occupation. Clinical distress scales were CWP-related symptoms such as fatigue severity, depression, level of stress response and the Korean version of the Fibromyalgia Impact Questionnaire (FIQ-K). The severity of fatigue was assessed by the Korean version of the Fatigue Severity Scale (FSS). ${ }^{10)}$ FSS consisted of 9 items asking fatigue severity on each different situation in the past 7 days. Each item was scored between 1 to 7 points, and summed to a score from 9 to 63 points. We used the Korean version of the Beck Depression Inventory (BDI) for measuring the severity of depression. ${ }^{11)}$ BDI had 21 questions asking severity of emotional, cognitional and physiological symptoms; responses were scored on a 0 to 3 points scale for each item. To evaluate the level of stress response, a Stress Response Inventory (SRI) was applied to the subjects. ${ }^{12)}$ SRI had four domains of 39 items (emotional, physical, cognitional and behavioral responses of subjects) and we measured levels of stress response in subjects by summation of the SRI score (score range, 0 to 156). We used the FIQ-K for evaluating the impact of CWP on daily living. ${ }^{13)}$ FIQ-K consisted of a physical function and nine FMS-related symptoms (feeling good, sick-leave, impact of fibromyalgia on work, pain, fatigue, morning tiredness, stiffness, anxiety, depression during the previous week). Physical function consisted of 11 items of everyday situations and was assessed by summing the total scores on a four point Likert scale for each item. FMS related nine symptoms were expressed using a visual analog scale (VAS).

\section{Diagnosis of Chronic Widespread Pain Patients}

The diagnosis of CWP was done after the patients had met the physicians at least 3 times. Physicians noted medical history (including psychosocial history) and physical examinations thoroughly, and ordered laboratory tests if necessary. Physicians concluded whether there was a cause that could explain CWP as the final disorder of CWP patients. The diagnosis of CWP was based on diagnostic criteria of a specific disease or syndrome and also physician's clinical decision. DSM-IV criteria were applied for diagnosing psychological disorders such as depression, panic disorder, anxiety, etc. FMS was diagnosed by ACR criteria. ${ }^{6}$ Those who complained of severe fatigue lasting for more than 6 months were diagnosed with chronic fatigue syndrome (CFS) or idiopathic chronic fatigue (ICF) by the case definition of CDC $(1988){ }^{14)}$

\section{Classification of Subjects}

After major problems associated with CWP were diagnosed by physicians, the subjects were classified into two different groups. One was the medically explained CWP group (ME) and the other was medically unexplained CWP group (MUE). ME was then reclassified into two subgroups, a psychological disorder group (PSY) and an organic disorder group (ORG) by their 
diagnosis. Most MUE patients were diagnosed as FMS, CFS, and ICF. If a subject had 2 more disorders for the possible cause of CWP and co-morbidities such as FMS and depression, physician decided the main cause of CWP clinically.

\section{Statistical Analysis}

We compared the socio-demographic and clinical distress scales among three groups (ORG, PSY, MUE). Clinical distress scales were FIQ-K, FSS, BDI, SRI, and the number of the tender

Table 1. Baseline data of the chronic widespread pain subjects $(\mathrm{n}=$ 109).

\begin{tabular}{|c|c|c|c|c|}
\hline & $\begin{array}{l}\text { MUE } \\
(\mathrm{n}=39)\end{array}$ & $\begin{array}{c}\text { ORG } \\
(n=25)\end{array}$ & $\begin{array}{c}\text { PSY } \\
(n=45)\end{array}$ & $\mathrm{P}$-value \\
\hline Age (y) & $46.3(14.8)$ & $52.0(12.7)$ & 50.4 (13.9) & 0.221 \\
\hline Female & $29(74.4)$ & $21(84.0)$ & 32 (71.1) & 0.483 \\
\hline BMI & 22.7 (2.5) & $23.5(3.7)$ & $22.8(2.5)$ & 0.558 \\
\hline Smoking status & & & & 0.013 \\
\hline Current smoker & $24(64.9)$ & 19 (79.2) & 27 (65.9) & \\
\hline Ex-smoker & $11(29.7)$ & $1(4.2)$ & $4(9.8)$ & \\
\hline Non-smoker & $2(5.4)$ & $4(16.7)$ & $10(24.4)$ & \\
\hline Alcohol & & & & 0.193 \\
\hline $\begin{array}{l}\text { Less than } \\
1 \text { time/mo }\end{array}$ & $27(96.4)$ & $18(85.7)$ & 31 (81.6) & \\
\hline $\begin{array}{l}\text { More than } \\
2-3 \text { times/mo }\end{array}$ & $1(3.6)$ & $3(14.3)$ & 7 (18.4) & \\
\hline Marital status & & & & 0.096 \\
\hline Married & $26(68.4)$ & $21(87.5)$ & 35 (85.4) & \\
\hline Unmarried & 12 (31.6) & $3(12.5)$ & $6(14.6)$ & \\
\hline Education & & & & 0.077 \\
\hline $\begin{array}{l}\text { High school } \\
\text { graduated or less }\end{array}$ & $14(36.8)$ & $12(50.0)$ & $25(62.5)$ & \\
\hline $\begin{array}{l}\text { College graduated } \\
\text { or more }\end{array}$ & $24(63.2)$ & $12(50.0)$ & 15 (37.5) & \\
\hline Occupation & & & & 0.438 \\
\hline Full time job & $20(52.6)$ & $15(60.0)$ & 19 (44.2) & \\
\hline Others & 18 (47.4) & $10(40.0)$ & 24 (55.8) & \\
\hline
\end{tabular}

Values are presented as mean (SD) or number (\%).

P-value $<0.05$ indicates a statistically significant difference.

MUE: medically unexplained, ORG: organic problem associated with CWP, PSY: psychological problems associated with CWP, CWP: chronic widespread pain, BMI: body mass index, SD: standard deviation. points over the entire body. FIQ-K among three groups were analyzed by the score of physical functioning and each score of the FMS (nine items) as well as the summation score of the FIQ-K (score of the physical functioning plus scores of the nine items). For comparison of categorical variables, we used $\chi^{2}$ analysis including non-parametric tests. Continuous variables were compared by one-way ANOVA and Tukey's post hoc test. To adjust for confounders, we used ANCOVA for comparing clinical distress scales among three groups. A P $<0.05$ was defined as statistically significant. Statistical analysis was performed using SPSS statistics version 18 software (PASW statistics 18, SPSS Inc., Chicago, IL, USA).

\section{RESULTS}

According to the physicians' diagnosis, 109 CWP patients were classified into 3 groups, 39 for MUE, 25 for ORG and 45 for PSY. Table 1 shows the socio-demographic characteristics of each group. In the MUE group, ex-smokers were significantly more frequent than in the other groups.

Table 2. Major problems associated with chronic widespread pain patients $(n=109)$.

\begin{tabular}{crlrlr}
\hline $\begin{array}{c}\text { MUE } \\
(\mathrm{n}=39)\end{array}$ & No. & \multicolumn{1}{c}{$\begin{array}{c}\text { ORG } \\
(\mathrm{n}=25)\end{array}$} & No. & $\begin{array}{c}\text { PSY } \\
(\mathrm{n}=45)\end{array}$ & No. \\
\hline FMS & 24 & Rheumatoid arthritis & 10 & Depression & 23 \\
CFS & 10 & Osteoarthritis & 6 & Stress/overwork & 6 \\
ICF & 5 & Gout & 1 & Panic disorder & 4 \\
& & Osteoporosis & 1 & Somatoform & 4 \\
& & & & disorder & \\
& & Thyroid cancer & 1 & Anxiety disorder & 4 \\
& & Stomach cancer & 1 & HwaByung & 4 \\
& Brain tumor & 1 & & \\
& Grave's disease & 1 & & \\
& Hypothyroidism & 1 & & \\
& Seizure & 1 & & \\
& Atopic dermatitis & 1 & & \\
\hline
\end{tabular}

MUE: medically unexplained, ORG: organic problem associated with CWP, PSY, psychological problem associated with CWP, CWP: chronic widespread pain, FMS: fibromyalgia syndrome, CFS: chronic fatigue syndrome, ICF: idiopathic chronic fatigue. 
Major problems associated with CWP patients were shown in Table 2. Thirty-nine patients of MUE were diagnosed as FMS $(n=24,61.5 \%)$, CFS $(n=15,25.5 \%)$, and ICF $(n=5,12.8 \%)$. In ORG, rheumatoid arthritis (RA; $\mathrm{n}=10,40 \%)$ was the most common medical problem followed by osteoarthritis (OA; $\mathrm{n}=6,24 \%)$, other musculoskeletal or metabolic diseases and malignancies. In PSY, depression $(n=23,53 \%)$ was the most common problem as well as stress and being overworked. Panic, anxiety and somatization disorders were also diagnoses for PSY.

Table 3 showed Clinical Distress Scales among three groups. Physical impairment scores were $15.3 \pm 7.9$ for MUE, $11.3 \pm$ 8.7 for ORG, and 14.8 \pm 8.1 for PSY but there was no difference among them $(\mathrm{P}=0.145)$. The numbers of days feeling good in the past seven days were significantly lower for MUE and PSY than ORG $(P=0.001)$. Work missed was not different among groups.
Tiredness was significantly higher in MUE than ORG and anxiety scores were higher in PSY than ORG. But, the ability to do work, pain, rest, stiffness and depression levels were not significantly different among groups. Comparing the FIQ-K scores of each group, MUE had a higher score than ORG group $(\mathrm{P}=0.003)$. MUE had a higher level of fatigue severity as assessed by FSS than PSY and ORG $(\mathrm{P}=0.007)$. BDI score was highest in PSY. MUE showed a higher stress score than ORG and more numbers of tender points than the other two groups $(\mathrm{P}<0.0001)$. As a result of multivariated analysis, MUE group showed significantly higher score in FIQ FSS, and SRI than ORG group. PSY group showed higher score in VAS anxiety scale, FIQ, BDI, and SRI than ORG group significantly. MUE had a higher number of tender points than the other two groups after mutlivariated analysis. Otherwise the number of days felt good last week was slightly higher in MUE

Table 3. Clinical distress scales of MUE, ORG, and PSY disorders associated with CWP.

\begin{tabular}{|c|c|c|c|c|c|c|c|}
\hline & \multicolumn{2}{|c|}{ MUE (n = 39) } & \multicolumn{2}{|c|}{ ORG $(\mathrm{n}=25)$} & \multicolumn{2}{|c|}{ PSY $(n=45)$} & \multirow{2}{*}{ P-value } \\
\hline & Mean & SD & Mean & SD & Mean & SD & \\
\hline \multicolumn{8}{|l|}{ FIQ-K } \\
\hline Physical impairment & 15.3 & 7.9 & 11.3 & 8.7 & 14.8 & 8.1 & 0.140 \\
\hline Feel good & 4.1 & 3.4 & $6.9^{*}$ & 2.3 & 5.7 & 2.8 & 0.001 \\
\hline Work missed & 1.2 & 2.4 & 0.1 & 0.4 & 0.7 & 2.4 & 0.160 \\
\hline Do work & 6.2 & 3.1 & 4.5 & 3.7 & 5.7 & 3.2 & 0.118 \\
\hline Pain & 6.2 & 2.9 & 4.4 & 3.4 & 5.4 & 3.3 & 0.088 \\
\hline Fatigue & $8.2^{*}$ & 1.8 & 6.3 & 3.0 & 7.2 & 2.8 & 0.025 \\
\hline Rested & 7.7 & 2.4 & 6.7 & 2.5 & 7.0 & 2.6 & 0.270 \\
\hline Stiffness & 7.2 & 2.5 & 5.6 & 3.0 & 5.9 & 3.2 & 0.076 \\
\hline Anxiety & $7.3^{*}$ & 2.4 & 5.3 & 3.3 & $7.3^{\dagger}$ & 2.5 & 0.007 \\
\hline Depression & 6.8 & 2.7 & 5.6 & 3.2 & 6.9 & 2.4 & 0.116 \\
\hline Sum. of FIQ-K & $72.4^{*}$ & 18.1 & 53.4 & 22.8 & 65.3 & 22.3 & 0.003 \\
\hline Fatigue severity scale & $48.6^{\ddagger}$ & 13.4 & 37.1 & 17.3 & 39.3 & 16.2 & 0.007 \\
\hline Beck depression inventory & 51.4 & 8.4 & 46.8 & 9.1 & $52.3^{\dagger}$ & 8.2 & 0.034 \\
\hline Stress response inventory & $103.6^{*}$ & 27.9 & 74.0 & 30.4 & $99.9^{\dagger}$ & 31.5 & $<0.0001$ \\
\hline NTPs & $11.3^{\ddagger}$ & 5.5 & 6.5 & 4.8 & 5.0 & 3.9 & $<0.0001$ \\
\hline
\end{tabular}

Data were analyzed by one-way ANOVA followed by Tukey post Hoc test. P-value $<0.05$ indicates statistical significance.

MUE: medically unexplained, ORG: organic problem associated with CWP, PSY: psychological problem associated with CWP, CWP: chronic widespread pain, SD: standard deviation, FIQ-K: Korean version of Fibromyalgia Impact Questionnaire, Sum. of FIQ-K: summation score of FIQ-K, NTPs: no. of tender points over the entire body.

*Significant difference between MUE and ORG group. ${ }^{\dagger}$ Significant difference between ORG and PSY groups. ${ }^{\ddagger}$ Significant difference between MUE and ORG, PSY groups. 

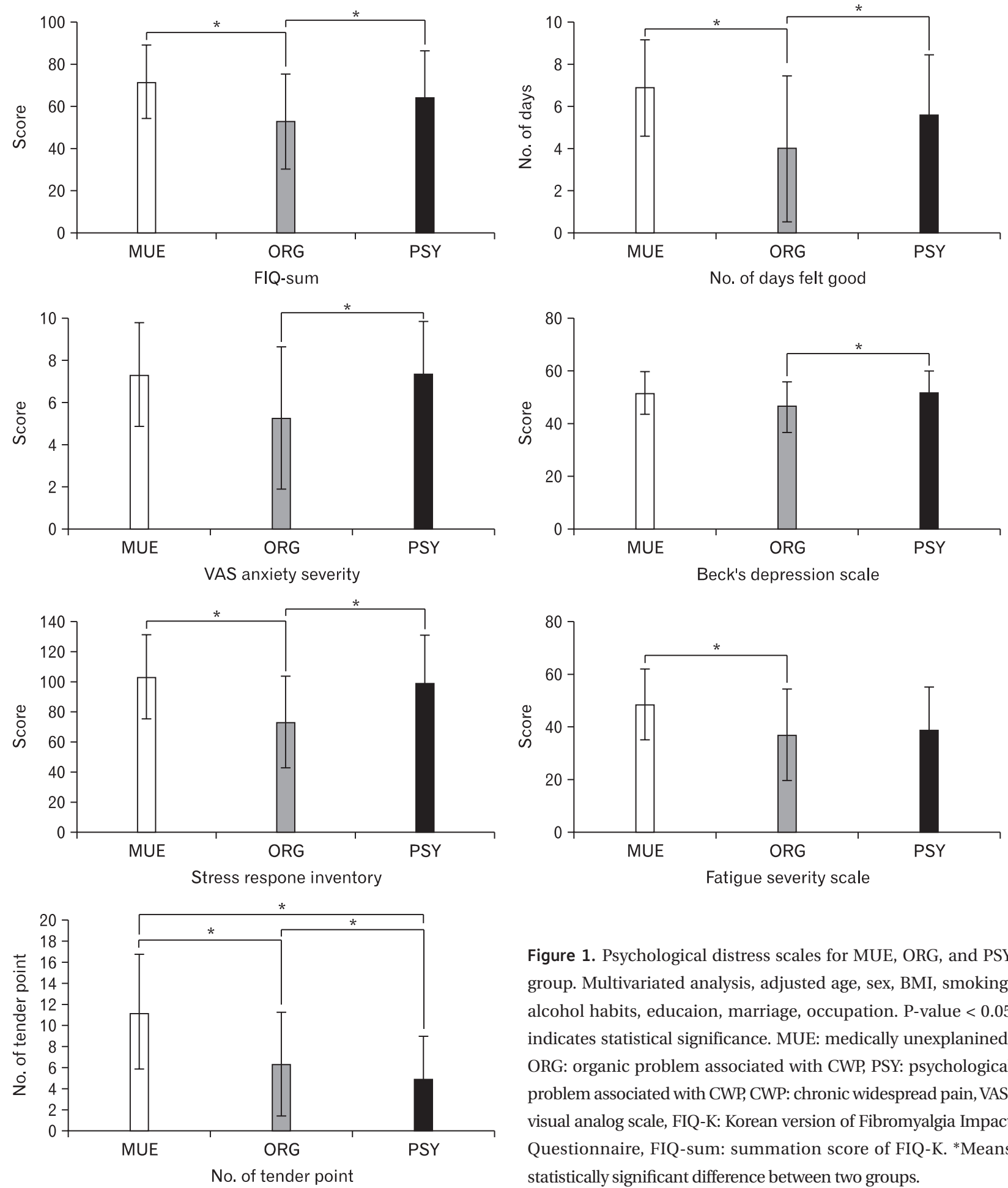

Figure 1. Psychological distress scales for MUE, ORG, and PSY group. Multivariated analysis, adjusted age, sex, BMI, smoking, alcohol habits, educaion, marriage, occupation. P-value $<0.05$ indicates statistical significance. MUE: medically unexplanined, ORG: organic problem associated with CWP, PSY: psychological problem associated with CWP, CWP: chronic widespread pain, VAS: visual analog scale, FIQ-K: Korean version of Fibromyalgia Impact Questionnaire, FIQ-sum: summation score of FIQ-K. *Means statistically significant difference between two groups.

group than other 2 groups. The results were presented in Figure 1.

\section{DISCUSSION}

It is known that patients with CWP suffer more psychological illness and often experience more disorders such as CFS, FMS, 
depression, and irritable bowel disease than those without CWP. ${ }^{15,16)}$ FMS and CFS are also well known disorders with CWP. ${ }^{17)}$ These syndromes have common complaints including severe fatigability, disability, chronic pain and depressive mood as well as a tendency to progress to a chronic state. ${ }^{18)}$ But neither biologic indicators nor specific diagnostic criteria exist to explain these syndromes. ${ }^{4)}$ We therefore conducted this study to search for differential clinical characteristics of MUE for which diagnoses and definitions are unclear between psychological illness and organic disorders.

Our research showed that patients with MUE had a higher level of fatigue, physical impairment and number of tender points on the body than ME, especially ORG but clinical distress symptoms such as severity of depression and level of stress were no different between MUE and PSY. MUE had differential clinical distress symptoms from ORG such as rheumatoid arthritis and osteoarthritis but similar with PSY. We suggest a few explanations for these similarities between MUE and PSY. First, MUE (FMS and CFS in our study) is not a single disorder but a spectrum of several disorders. Bradley ${ }^{19)}$ and Hudson et al. ${ }^{20)}$ had suggested that ten types of mental illnesses including major depression, generalized anxiety disorder and four kinds of medical conditions including FMS, IBS, migraine and cataplexy as referred as affective spectrum disorders. Additionally, another study showed that lifetime prevalence of major depression in FMS patients was significantly higher than in the general population and as with depression, FMS is accompanied by similar symptoms including various functional disabilities, depressive moods, memory impairment, vulnerability to stress and autonomic nervous system dysfunction. Therefore, both disorders have been claimed to have the same underlying mechanism. ${ }^{21)}$ Second, in patients with CFS or FMS, antidepressants can relieve their pain regardless of the mood of patients. ${ }^{22,23)}$ Thus, medically unexplained syndromes like FMS and CFS are likely to have a similar disease mechanism including depression and somatization disorders. Third, the prevalence of depression in patients with FMS or CFS is higher than in the general population. In a Finnish study, the prevalence of depression was much higher in FMS patients than those without FMS (56\% vs. 17\%). ${ }^{24)}$ Raphael et el. ${ }^{25)}$ also reported that women with FMS had a $69.8 \%$ chance of life-time prevalence of depression compared to $34.3 \%$ of women with no FMS in a community-based study. Lars et al. reported that $20 \%$ and
$10 \%$ of CWP complaints were due to rheumatoid arthritis and degenerative arthritis, respectively. ${ }^{26)}$

In our study, ORG of CWP patients was 22.9\% (25/109), $9.2 \%(10 / 109)$ for RA and $5.5 \%$ for OA (6/109). These low percentages of RA and OA for CWP in our study were thought to be due to the difference of study subjects visiting our center and further research is needed in Korea for clarification.

Our study showed that MUE tended to have poorer physical functioning, higher levels of stress response and fatigue severity than PSY. FMS had a number of associated symptoms such as chronic fatigue, irritable bowel and chronic pelvic pain while patients with multiple conditions had a poor quality of life, more severe functional disabilities and depressive moods. ${ }^{27,28)}$ Although co-morbidity conditions of MUE were not evaluated in this study, they were more likely to have associated psychological diseases which give rise to the functional disability and psychological symptoms.

MUE showed more severe depressed moods, higher levels of stress response and severe fatigue than the ORG. And functional impairment due to pain was more severe in MUE. These results were consistent with previous studies. ${ }^{10,29)}$

The number of tender points over the entire body was significantly higher in MUE than in ME. Because MUE included FMS, the result was not surprising. These results were also consistent with previous studies showing that FMS had more tender points and also higher distress such as fatigue as well as impairment of physical functioning. ${ }^{18,30)}$ Additionally, Yunus ${ }^{31)}$ suggested a concept of central sensitization (CS) that pain in FMS is related to CS and the pathophysiological mechanism of CS is associated with activation of peripheral nociceptive $\mathrm{C}$ fibers in chronic pain by several neurochemicals (substances $\mathrm{P}$, serotonin and bradykinin). In practice, CS can be checked by tender points on the body indirectly. Thus, our results that MUE had increased numbers of tender points on the body may be consistent with the aforementioned hypothesis of CS.

There are some limitations of our study. First, the major problem associated with CWP of subjects was determined by only a single disorder. Most of the patients with CWP had several diseases and it is difficult to confirm whether one or two specific diseases or a syndrome were the major cause of CWP. ${ }^{32)}$ Thus, we had a difficulty in differentiating between MUE and PSY because most MUE patients had psychological co-morbidities 
such as depression, sleep disturbances etc. However, in our study, physicians interviewed the patients more than three times and performed CWP-related laboratory tests, if necessary and then decided only a major cause explaining CWP. Thus we think that this limitation is not big enough to affect our results but further studies will be necessary in the future. Second, the study results can't represent the characteristics of all CWP patients because the subjects were patients visiting the department of a university hospital. Third, the number of study subjects was relatively small so that some clinical characteristics of MUE were unlikely to be significantly different from ME.

Despite these limitations, we found MUE had some differential clinical distress symptoms from ME, especially ORG and our study was the first investigation for clinical characteristics of medically unexplained CWP patients in Korea. In the future, further studies are needed to determine whether the various symptoms presented in patients with medically unexplained chronic widespread pain might explain the pathophysiology of the pain mechanisms.

\section{REFERENCES}

1. Bergman S, Herrstrom P, Hogstrom K, Petersson IF, Svensson B, Jacobsson LT. Chronic musculoskeletal pain, prevalence rates, and sociodemographic associations in a Swedish population study. J Rheumatol 2001;28:1369-77.

2. Wolfe F, Ross K, Anderson J, Russell IJ, Hebert L. The prevalence and characteristics of fibromyalgia in the general population. Arthritis Rheum 1995;38:19-28.

3. McBeth J, Macfarlane GJ, Hunt IM, Silman AJ. Risk factors for persistent chronic widespread pain: a community-based study. Rheumatology (Oxford) 2001;40:95-101.

4. Papageorgiou AC, Silman AJ, Macfarlane GJ. Chronic widespread pain in the population: a seven year follow up study. Ann Rheum Dis 2002;61:1071-4.

5. Kato K, Sullivan PF, Evengård B, Pedersen NL. Chronic widespread pain and its comorbidities: a population-based study. Arch Intern Med 2006;166:1649-54.

6. Wolfe F, Smythe HA, Yunus MB, Bennett RM, Bombardier C, Goldenberg DL, et al. The American College of Rheumatology 1990 Criteria for the Classification of Fibromyalgia: report of the Multicenter Criteria Committee. Arthritis Rheum 1990;33:160-72.

7. Gran JT. The epidemiology of chronic generalized musculoskeletal pain. Best Pract Res Clin Rheumatol 2003;17:547-61.

8. MacFarlane GJ, Croft PR, Schollum J, Silman AJ. Widespread pain: is an improved classification possible? J Rheumatol 1996;23:1628-32.

9. Bliddal H, Danneskiold-Samsoe B. Chronic widespread pain in the spectrum of rheumatological diseases. Best Pract Res Clin Rheumatol 2007;21:391-402.

10. Jung KI, Song $\mathrm{CH}$. Clinical usefulness of fatigue severity scale for patients with fatigue, and anxiety or depression. Korean J Psychosom Med 2001;9:164-73.

11. Hahn HM, Yum TH, Shin YW, Kim KH, Yoon DJ, Chung KJ. A standardization study of beck depression inventory in Korea.J Korean Neuropsychiatr Assoc 1986;25:487-502.

12. Koh KB, ParkJK, Kim CH, Cho S. Development of the stress response inventory and its application in clinical practice. Psychosom Med 2001;63:668-78.

13. Kim YA, Lee SS, Park K. Validation of a Korean version of the Fibromyalgia Impact Questionnaire. J Korean Med Sci 2002;17:220-4.

14. Fukuda K, Straus SE, Hickie I, Sharpe MC, Dobbins JG, Komaroff A. The chronic fatigue syndrome: a comprehensive approach to its definition and study. International Chronic Fatigue Syndrome Study Group. Ann Intern Med 1994;121: 953-9.

15. Benjamin S, Morris S, McBeth J, Macfarlane GJ, Silman AJ. The association between chronic widespread pain and mental disorder: a population-based study. Arthritis Rheum 2000;43:561-7.

16. McBeth J, Jones K. Epidemiology of chronic musculoskeletal pain. Best Pract Res Clin Rheumatol 2007;21:403-25.

17. Aaron LA, Burke MM, Buchwald D. Overlapping conditions among patients with chronic fatigue syndrome, fibromyalgia, and temporomandibular disorder. Arch Intern Med 2000;160:221-7.

18. Clauw DJ, Crofford LJ. Chronic widespread pain and fibromyalgia: what we know, and what we need to know. Best Pract Res Clin Rheumatol 2003;17:685-701.

19. Bradley LA. Pathophysiologic mechanisms of fibromyalgia 
and its related disorders. J Clin Psychiatry 2008;69 Suppl 2:6-

13.

20. Hudson JI, Mangweth B, Pope HG Jr, De Col C, Hausmann A, Gutweniger S, et al. Family study of affective spectrum disorder. Arch Gen Psychiatry 2003;60:170-7.

21. Pae CU, Luyten P, Marks DM, Han C, Park SH, Patkar AA, et al. The relationship between fibromyalgia and major depressive disorder: a comprehensive review. Curr Med Res Opin 2008;24:2359-71.

22. Anderberg UM, Marteinsdottir I, von Knorring L. Citalopram in patients with fibromyalgia: a randomized, doubleblind, placebo-controlled study. Eur J Pain 2000;4:27-35.

23. Arnold LM, Lu Y, Crofford LJ, Wohlreich M, Detke MJ, Iyengar $S$, et al. A double-blind, multicenter trial comparing duloxetine with placebo in the treatment of fibromyalgia patients with or without major depressive disorder. Arthritis Rheum 2004;50:2974-84.

24. Makela M, Heliovaara M. Prevalence of primary fibromyalgia in the Finnish population. BMJ 1991;303:216-9.

25. Raphael KG, Janal MN, Nayak S, Schwartz JE, Gallagher RM. Psychiatric comorbidities in a community sample of women with fibromyalgia. Pain 2006;124:117-25.

26. White KP, Nielson WR, Harth M, Ostbye T, Speechley M. Chronic widespread musculoskeletal pain with or without fibromyalgia: psychological distress in a representative community adult sample. J Rheumatol 2002;29:588-94.

27. Epstein SA, Kay G, Clauw D, Heaton R, Klein D, Krupp L, et al. Psychiatric disorders in patients with fibromyalgia: a multicenter investigation. Psychosomatics 1999;40:57-63.

28. Aaron LA, Buchwald D. Chronic diffuse musculoskeletal pain, fibromyalgia and co-morbid unexplained clinical conditions. Best Pract Res Clin Rheumatol 2003;17:563-74.

29. Gormsen L, Rosenberg R, Bach FW, Jensen TS. Depression, anxiety, health-related quality of life and pain in patients with chronic fibromyalgia and neuropathic pain. Eur J Pain 2010;14:127.e1-8.

30. Lundberg G, Gerdle B. Tender point scores and their relations to signs of mobility, symptoms, and disability in female home care personnel and the prevalence of fibromyalgia syndrome. J Rheumatol 2002;29:603-13.

31. Yunus MB. Role of central sensitization in symptoms beyond muscle pain, and the evaluation of a patient with widespread pain. Best Pract Res Clin Rheumatol 2007;21:481-97.

32. Macfarlane GJ, Morris S, Hunt IM, Benjamin S, McBeth J, Papageorgiou AC, et al. Chronic widespread pain in the community: the influence of psychological symptoms and mental disorder on healthcare seeking behavior. J Rheumatol 1999;26:413-9. 